\title{
LINKING FORMS AND MAPS OF ODD PRIME ORDER
}

BY

\author{
J. P. ALEXANDER, G. C. HAMRICK AND J. W. $\operatorname{VICK}\left({ }^{1}\right)$
}

ABSTRACT. A differentiable orientation preserving map of odd prime period on a closed oriented differentiable manifold gives rise to two invariants taking values in a Witt group of bilinear forms. One is globally defined in terms of the rational cohomology of the manifold and the other is locally defined in terms of the fixed point set and its normal bundle. We show that these two invariants are, in fact, equal and apply this result to relate the structure of the manifold to that of the fixed point set and the quotient space.

Let $M^{4 n}$ be a closed oriented differentiable manifold and $T$ be an orientation preserving diffeomorphism of $M$ of odd prime period $p$. Using the representation $T^{*}$ of $\mathrm{Z}_{p}$ in $H^{2 n}(M ; Q)$, Conner and Raymond [8] defined a torsion element $q(T, M)$ in the rational Witt ring $W(Q)$. This is an invariant of the equivariant cobordism class of the action and vanishes if the action is fixed point free; hence it may be expressed in terms of the fixed point set and the action of $Z_{p}$ in its normal bundle. Conner and Raymond gave such an expression for $p=3$. In [4] we announced a formula for all odd primes. The purpose of this paper is to give the details of the proofs along with some additional background and applications.

An essential factor in the proof is the relationship between rational forms and forms on finite abelian groups. This approach has also been effective in dealing with other problems [1] , [2]. Here we use it to relate the peripheral invariant for a compact bounding ( $4 n-1)$-dimensional manifold $V$, an element of $W(Q)$ defined in [8], to the linking form on the torsion subgroup of $H^{2 n}(V ; Z)$.

$\$ 1$ contains the requisite algebraic material on bilinear forms and Witt groups. In the second section the peripheral invariant is defined and is shown to correspond to the negative of the linking form in the Witt group of finite forms. The formula expressing $q(T, M)$ in terms of the fixed point data is established in $\S 3$ and applied to prove the following:

(3.3) If $T^{*}$ is the identity on $H^{2 n}(M ; Q)$, then

(a) for $p \equiv 3(\bmod 4), \operatorname{sgn} M \equiv \operatorname{sgn} F(\bmod 4)$;

Received by the editors May 5, 1975.

AMS (MOS) subject classifications (1970). Primary 55C35, 57D99; Secondary 15 A63.

(1) This research was supported by NSF Grant GP-43775.

Copyright $\odot$ 1976, American Mathematical Society 
(b) for $p \equiv 1(\bmod 4)$, $\operatorname{sgn} M \equiv \operatorname{sgn} F \equiv \operatorname{sgn} F_{0}(\bmod 2) ;$ and

(3.6) $\operatorname{sgn} M-p \cdot \operatorname{sgn}(M / T)= \begin{cases}\operatorname{sgn} F \cdot(1-p) \bmod 8, & \text { if } p \equiv 3 \bmod 4, \\ \operatorname{sgn} F_{1}(5-p) \bmod 8, & \text { if } p \equiv 1 \bmod 8, \\ \operatorname{sgn} F_{0}(1-p) \bmod 8, & \text { if } p \equiv 5 \bmod 8,\end{cases}$ where $F_{0}$ and $F_{1}$ are prescribed disjoint closed subsets of the fixed point set $F$ with $F_{0} \cup F_{1}=F$.

In [5] we used the Atiyah-Singer Index Theorem to show that (3.3) (a) actually holds for any diffeomorphism $T$ of odd period having $T^{*}=$ identity on $H^{2 n}(M ; Q)$.

I. Bilinear forms. In this section we collect a number of techniques which are useful in studying rational bilinear forms. A symmetric form $(V, f)$ consists of a bilinear map $f: V \times V \rightarrow M$ such that $f\left(v, v^{\prime}\right)=f\left(v^{\prime}, v\right)$ for all $v, v^{\prime} \in V$, where $M$ may be $Q, \mathrm{Z}$ or $Q / \mathrm{Z}$ and the corresponding $V$ will be a finite dimensional rational vector space, a finitely generated free abelian group or a finite abelian group. A form is nonsingular if the adjoint map $\hat{f}: V \rightarrow \operatorname{Hom}(V, M)$ given by $\hat{f}(v)\left[v^{\prime}\right]=f\left(v, v^{\prime}\right)$ is an isomorphism. If $A \subseteq V$, denote by $A^{\perp}$ the set of all elements that annihilate $A$,

$$
A^{\perp}=\{v \in V \mid f(v, a)=0 \text { for all } a \in A\} .
$$

A form $(V, f)$ is called metabolic if there exists a subgroup $U \subseteq V$ such that $U=$ $U^{\perp}$. Two forms $(V, f)$ and $\left(V^{\prime}, f^{\prime}\right)$ are isomorphic if there exists an isomorphism $\varphi: V \rightarrow V^{\prime}$ such that $f^{\prime}(\varphi(v), \varphi(w))=f(v, w)$ for all $v, w \in V$. The orthogonal direct sum of $(V, f)$ and $\left(V^{\prime}, f^{\prime}\right)$ is defined in the obvious way and denoted by $(V, f) \perp\left(V^{\prime}, f^{\prime}\right)$.

Define an equivalence relation on nonsingular forms by setting $(V, f) \sim$ $\left(V^{\prime}, f^{\prime}\right)$ if there exist metabolic forms $(U, g)$ and $\left(U^{\prime}, g^{\prime}\right)$ such that $(V, f) \perp(U, g)$ is isomorphic to $\left(V^{\prime}, f^{\prime}\right) \perp\left(U^{\prime}, g^{\prime}\right)$. The set of equivalence classes forms a group $W(M)$ with group operation given by orthogonal direct sum. $W(M)$ is the Witt group of rational, integral or finite forms as $M=Q, \mathrm{Z}$ or $Q / \mathrm{Z}$, respectively.

(1.1) LEMMA. If $V$ is metabolic and $U \subseteq V$ is a submodule with $U \subseteq U^{\perp}$, then there exists a submodule $\bar{U}$ with $U \subseteq \bar{U}=\bar{U}^{\perp}$.

Proof. We prove this for rational or finite forms. The result for integral forms is a consequence of the rational case. First note that if $A$ and $B$ are submodules of $V$ then $(A+B)^{\perp}=A^{\perp} \cap B^{\perp}$ and $(A \cap B)^{\perp}=A^{\perp}+B^{\perp}$. Since $V$ is metabolic there exists a submodule $S \subseteq V$ with $S=S^{\perp}$. Let $\bar{U}=U^{\perp} \cap(U+S)$. Then $U \subseteq \bar{U}$ and 


$$
\begin{aligned}
\bar{U}^{\perp} & =\left(U^{\perp}\right)^{\perp}+(U+S)^{\perp}=U+\left(U^{\perp} \cap S^{\perp}\right) \\
& =\left(U^{\perp} \cap U\right)+\left(U^{\perp} \cap S^{\perp}\right)=U^{\perp} \cap\left(U+S^{\perp}\right) \\
& =U^{\perp} \cap(U+S)=\bar{U} . \quad
\end{aligned}
$$

(1.2) Lemma. [ $V, f]=0$ in $W(\dot{M})$ if and only if $(V, f)$ is metabolic.

Proof. If $[V, f]=0$ there exist metabolic modules $W$ and $W^{\prime}$ such that $V \perp W \approx W^{\prime}$. Let $U \subseteq W$ be a submodule such that $U=U^{\perp}$ in $W$. By (1.1) there is a $\bar{U} \subseteq V \perp W$ such that $U \subseteq \bar{U}=\bar{U}^{\perp}$ in $V \perp W$. Now if $(v, w) \in \bar{U}$ then $w$ annihilates $U$, hence $w \in U$. Since $U \subseteq \bar{U}$ it follows that $(v, 0) \in \bar{U}$. So if $S=$ $V \cap \bar{U}$ then $S \subseteq S^{\perp}$ in $V$. On the other hand if $v \in S^{\perp}$ then $(v, 0)$ annihilates $S$ and $U$, hence it annihilates $\bar{U}$. Thus $S^{\perp} \subseteq S$ and $V$ is metabolic. The other implication is trivial.

(1.3) Lemma. If $[V, f] \in W(M)$ and $U \subseteq U^{\perp}, \subseteq V$ are submodules, then $[V, f]=\left[U^{\perp} / U, f^{\prime}\right]$ where $f^{\prime}$ is the form induced by $f$.

Proof. We must show that $(V, f) \perp\left(U^{1} / U,-f^{\prime}\right)$ is metabolic. Let $\theta$ : $U^{\perp} \rightarrow V \perp U^{\perp} / U$ be given by $\theta(v)=(v, v+U)$. Then

$$
\begin{aligned}
\left(f \perp\left(-f^{\prime}\right)\right)(\theta(u), \theta(v)) & =f(u, v)-f^{\prime}(u+U, v+U) \\
& =f(u, v)-f(u, v)=0 .
\end{aligned}
$$

Consequently $\theta\left(U^{\perp}\right)$ is contained in its annihilator. Suppose $x \in \theta\left(U^{\perp}\right)^{\perp}$ and write $x=\left(v, v^{\prime}+U\right) . x$ annihilates everything of the form $(u, 0+U)$ where $u \in U$, hence $f(v, u)=0$ and $v \in U^{\perp}$. For any $u \in U^{\perp}$,

$$
0=\left(f \perp\left(-f^{\prime}\right)\right)(x, \theta(u))=f(v, u)-f\left(v^{\prime}, u\right)=f\left(v-v^{\prime}, u\right) .
$$

So the element $v-v^{\prime}$ in $V$ lies in $\left(U^{\perp}\right)^{\perp}=U$ and $v \equiv v^{\prime} \bmod U$. Thus $x \in$ $\theta\left(U^{\perp}\right)$ and $\theta\left(U^{\perp}\right)$ is equal to its own annihilator.

Suppose $(V, f)$ is a rational form. An integral lattice $L \subseteq V$ is a free Zmodule such that $L \otimes Q=V$ and $f(x, y) \in \mathbf{Z}$ for all $x, y \in L$. Let $L^{+}=\{x \in$ $V \mid f(x, y) \in \mathbf{Z}$ for all $y \in L\}$, the dual lattice for $L . L^{+}$is isomorphic to $\operatorname{Hom}(L, Z)$, and since $f$ is nonsingular, the induced map $\hat{f}: L \rightarrow \operatorname{Hom}(L, Z)$ is compatible with the inclusion $L \subseteq L^{+} . L^{+} / L$ is a finite abelian group which carries a nonsingular form given by $\bar{f}(x+L, y+L)=\rho(f(x, y))$ where $\rho: Q \rightarrow$ $Q / Z$ is reduction.

(1.4) Lemma. The correspondence $(V, f) \mapsto\left(L^{+} / L, \bar{f}\right)$ defines a homomorphism $\partial: W(Q) \rightarrow W(Q / \mathbf{Z})$.

Proof. We must show that the Witt class of the finite form is independent of the lattice chosen and that $\partial$ vanishes on metabolic modules. If $L_{1}$ and $L_{2}$ are lattices in $V$, we can find a lattice $L_{1} \cap L_{2}$ which is contained in both. So we assume that $L_{1} \subseteq L_{2}$. It follows that $L_{1} \subseteq L_{2} \subseteq L_{2}^{+} \subseteq L_{1}^{+}$. If $G=L_{1}^{+} / L_{1}$ 
and $H=L_{2} / L_{1}$, the annihilator of $H$ in $G$ is $H^{\perp}=L_{2}^{+} / L_{1}$. Now $H \subseteq H^{\perp}$ so by (1.3) the form on $G=L_{1}^{+} / L_{1}$ is Witt equivalent to the form on $H^{\perp} / H \simeq L_{2}^{+} / L_{2}$. Hence $\partial[V, f]$ is independent of the choice of lattice.

Now suppose $(V, f)$ is metabolic, so there exists a subspace $U=U^{\perp} \subseteq V$. If $L$ is an integral lattice in $V$ then $U \cap L^{+}$is a summand of $L^{+}$. Note that

$$
\left(U \cap L^{+}\right)^{\perp}=U^{\perp}+\left(L^{+}\right)^{\perp}=U^{\perp}=U \text { since }\left(L^{+}\right)^{\perp}=0 \text {. }
$$

Furthermore, $\left(U \cap L^{+}\right)^{+}=U+L$. It is clear that any element of $U+L$ gives integer values on $U \cap L^{+}$. Conversely, suppose $x$ gives integer values on $U \cap L^{+}$ and write $L^{+}=\left(U \cap L^{+}\right) \oplus K$. We can find $y$ that annihilates $U \cap L^{+}$and agrees with $x$ on $K$. Then $x=y+(x-y)$ expresses $x$ as an element of $U+L$.

Consider the subgroup $H=\left(\left(U \cap L^{+}\right)+L\right) / L \subseteq L^{+} / L . H$ is clearly selfannihilating. If $z+L \in H^{\perp}$ then $z$ is integer valued on $U \cap L^{+}$, hence $z \in$ $U+L$ and $z+L \in H$. Therefore $H=H^{\perp}$ and $\partial[V, f]$ is metabolic.

(1.5) THEOREM ([9]). The sequence

$$
0 \rightarrow W(\mathrm{Z}) \stackrel{i}{\longrightarrow} w(Q) \stackrel{\partial}{\longrightarrow} w(Q / \mathrm{Z}) \rightarrow 0
$$

is exact, where $i[V, f]=[V \otimes Q, f \otimes 1]$.

REMARK. A similar theorem, most elegantly proven, appears in Milnor and Husemoller [9]. Their proof obtains as a by-product the much deeper fact that $W(Z) \approx \mathbf{Z}$ via the signature and thus the sequence splits. Our approach differs in that we employ finite forms which will be the key to our topological applications in the next section.

Proof. If $\partial[V, f]=0$, then the finite form on $L^{+} / L$ is metabolic. Let $H \subseteq L^{+} / L$ be a subgroup with $H=H^{\perp}$. Define $K=\left\{x \in L^{+} \mid x+L \in H\right\}$. If $x \in K^{+}$then $x+L \in H$ which implies that $x \in K$. Similarly $K \subseteq K^{+}$, so $K=$ $K^{+}$. Hence the form $f$ restricted to the lattice $K$ is integrally nonsingular and $[V, f]=\left[K \otimes Q,\left.f\right|_{K} \otimes 1\right]$ is in the image of $i$. It is clear that $\partial \circ i=0$ and $i$ is a monomorphism.

To show that $\partial$ is onto we will compute the structure of $W(Q / Z)$. For a prime $p$ let $G_{(p)}$ denote the $p$-primary part of $G$. A form on $G$ splits canonically into its primary parts $G=G_{(2)} \perp G_{(3)} \perp \cdots \perp G_{(p)} \perp \cdots$ where only finitely many terms are nonzero.

(1.6) LEMMA. $[G]=0$ in $W(Q / Z)$ if and only if $\left[G_{(p)}\right]=0$ for all primes $p$.

Proof. Clearly $\left[G_{(p)}\right]=0$ for all primes $p$ implies $[G]=0$. So suppose $[G]=0$. Then by (1.2), $G$ is metabolic. Let $K \subseteq G$ be a subgroup with $K=$ $K^{\perp}$, hence also having $|K|^{2}=|G|$. If $p^{n}$ is the highest power of $p$ dividing $|K|$, 
then $K_{(p)}$ is a self-annihilating subgroup of $G_{(p)}$ and

$$
\left|K_{(p)}\right|^{2}=p^{2 n}=\left|G_{(p)}\right|
$$

Hence $\left[G_{(p)}\right]=0$ for each prime $p$.

If $F_{p}$ denotes the field with $p$ elements, $p$ a prime, the group homomorphism $F_{p} \rightarrow Q / Z$ sending 1 to $1 / p$ identifies $F_{p}$ additively with the subgroup of order $p$ in $Q / Z$. A nonsingular bilinear form on a finite dimensional $F_{p}$ vector space may thus be viewed as a finite group form. This defines a homomorphism $\psi_{p}: W\left(F_{p}\right) \rightarrow W(Q / Z)$ where $W\left(F_{p}\right)$ is the Witt group [9].

(1.7) THEOREM. $\psi=\bigoplus_{p} \psi_{p}: \bigoplus_{p} W\left(F_{p}\right) \rightarrow W(Q / Z)$ is an isomorphism.

Proof. If $(V, f)$ is a form over $F_{p}$ with $\psi_{p}([V])=0$, then $V$ contains a subgroup $U$ with $U=U^{\perp}$. This $U$ is also an $F_{p}$-subspace with $\operatorname{dim} U=1 / 2 \operatorname{dim} V$. Hence $[V]=0$ in $W\left(F_{p}\right)$ and $\psi_{p}$ is monic. It then follows from (1.6) that $\psi$ is monic.

Let $(G, f)$ be a form on a group $G$ of exponent $p^{n}$. If $n=1,(G, f)$ is in the image of $\psi_{p}$. If $n \geqslant 1$, let $K=p^{n-1} G$. Then for $x, y \in K, x=p^{n-1} \bar{x}$, $y=p^{n-1} \bar{y}$ and $f(x, y)=p^{2 n-2} f(\bar{x}, \bar{y})=0$ in $Q / Z$. So $K \subset K^{\perp}$ and $(G, f)$ is Witt equivalent to $\left(K^{1} / K, \bar{f}\right)$, a form on a group of exponent less than $p^{n}$. Proceeding inductively, we see that $G$ is Witt equivalent to a form on a group of exponent $p$ and this form is in the image of $\psi_{p}$. We conclude that $\psi$ is onto.

Recall [9] the structure of $W\left(F_{p}\right)$ is given by

(i) for $p=2, W\left(F_{2}\right) \simeq Z_{2}$, generated by $\langle 1\rangle_{2}$;

(ii) for $p \equiv 3 \bmod 4, W\left(F_{p}\right) \simeq Z_{4}$, generated by $\langle 1\rangle_{p}$;

(iii) for $p \equiv 1 \bmod 4, W\left(F_{p}\right) \simeq Z_{2} \oplus Z_{2}$ with generating set $\langle 1\rangle_{p}$ and $\langle\alpha\rangle_{p}$ where $\alpha$ is a nonsquare $\bmod p$.

To explain this notation, let $Z_{n}$ be the cyclic group of order $n$ and let $a$ be an integer relatively prime to $n$. Then $\langle a\rangle_{n}$ denotes the form $\left(Z_{n}, f\right)$ where $f(1,1)=a / n$. Note that if $b$ is relatively prime to $n$ and $b$ is a square $\bmod n$, then $\langle a b\rangle_{n}$ is isomorphic to $\langle a\rangle_{n}$. If $a$ and $b$ are relatively prime integers, then $\langle 1\rangle_{a b}=\langle a\rangle_{b}+\langle b\rangle_{a}$ in $W(Q / Z)$. Note that $\langle 1\rangle_{a b}=\partial(a b)$ where $(a b)$ denotes the 1-dimensional rational form with matrix $(a b)$.

As a consequence of the structure of $W(Q / Z)$, it is sufficient to show that any finite form $\langle k\rangle_{n}$ is in the image of $\partial$ to conclude that $\partial$ is an epimorphism. We will construct a matrix for a rational form $V$ with $\partial(V)=\langle k\rangle_{n}$. This employs an algorithm due to Wall [10].

Assuming that $(n, k)=1$ apply the Euclidean algorithm to $n$ and $k=k_{0}$ : 


$$
\begin{aligned}
n & =\alpha_{0} k_{0}+k_{1}, \\
k_{0} & =\alpha_{1} k_{1}+k_{2}, \\
& \vdots \\
k_{m-1} & =\alpha_{m} k_{m}+1, \\
k_{m} & =\alpha_{m+1} \cdot 1+0 .
\end{aligned}
$$

Consider the rational form given by the diagonal matrix

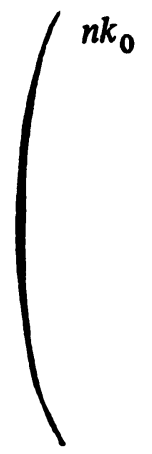

$-k_{0} k_{1}$

$$
k_{1} k_{2}
$$

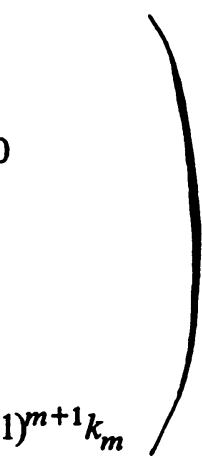

Since $\left(k_{i}, k_{i+1}\right)=1$ for each $i$ we use the previous observation to conclude that the image under $\partial$ of this form is

$$
\left\langle k_{0}\right\rangle_{n}+\langle n\rangle_{k_{0}}-\left\langle k_{1}\right\rangle_{k_{0}}-\left\langle k_{0}\right\rangle_{k_{1}}+\left\langle k_{2}\right\rangle_{k_{1}}+\left\langle k_{1}\right\rangle_{k_{2}}-\cdots
$$

But since $n \equiv k_{1}\left(\bmod k_{0}\right), k_{0} \equiv k_{2}\left(\bmod k_{1}\right)$, etc., this is just $\left\langle k_{0}\right\rangle_{n}=\langle k\rangle_{n}$ in $W(Q / Z)$. We conclude that $\partial$ is onto and the proof of $(1.5)$ is complete.

Note that the determinant of the matrix $(1.8)$ is $\pm n\left(k_{0}^{2} k_{1}^{2} \cdots k_{m}^{2}\right)$. If we wished to have a nondegenerate integral form that gave $\langle k\rangle_{n}$ precisely rather than up to Witt equivalence, we could perform simultaneous row and column operations on (1.8) to produce the matrix

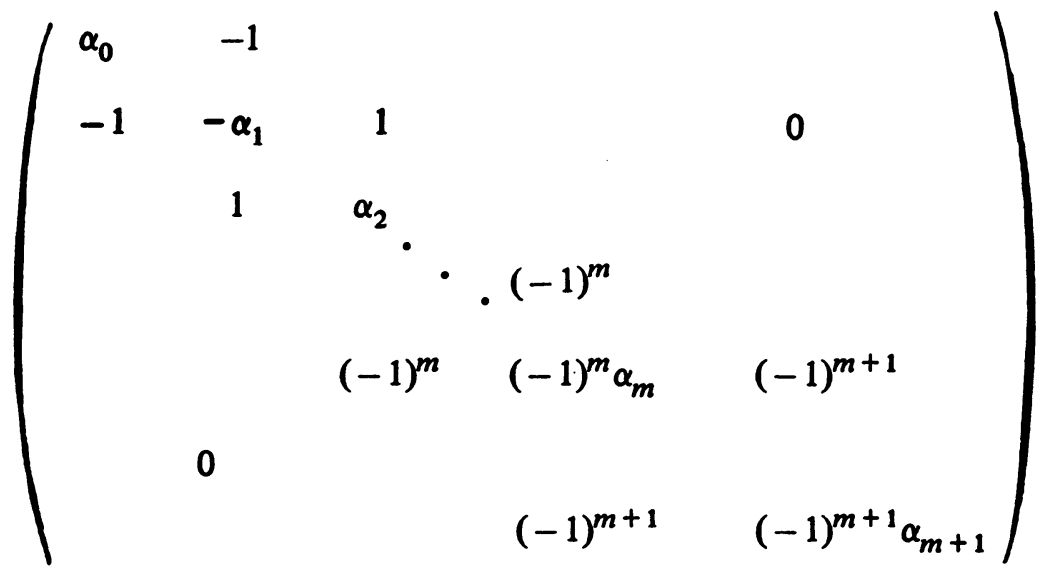

which has determinant $n$. 
II. The peripheral invariant and linking forms. Suppose $B^{4 n}$ is a compact oriented differentiable manifold and $M=\partial B$. If $V$ denotes the image of the homomorphism

$$
i^{*}: H^{2 n}(B, \partial B ; Q) \rightarrow H^{2 n}(B ; Q)
$$

there is a symmetric nonsingular bilinear form $f: V \times V \rightarrow Q$ given by

$$
f\left(i^{*}(x), i^{*}(y)\right)=\left\langle x \cup i^{*} y,[B, \partial B]\right\rangle \in Q
$$

This form represents an element $w(B)$ in $W(Q)$ whose signature we denote by $\operatorname{sgn}(B)$. Conner and Raymond [8] defined the peripheral invariant of $M$ to be

$$
\operatorname{per}(M)=w(B)-\operatorname{sgn}(B) \cdot 1 \in W(Q)
$$

for any bounding $(4 n-1)$-manifold $M$. Here 1 denotes the multiplicative identity in $W(Q)$.

To see that this is independent of the choice of $B$, suppose that $\bar{B}^{4 n}$ is another compact oriented differentiable manifold with $\partial \bar{B}^{4 n}=M$. Then $B \cup_{M}$ $-\bar{B}$ is closed, oriented and

$$
\begin{aligned}
0=w\left(B \cup \cup_{M}-\bar{B}\right) & -\operatorname{sgn}\left(B \cup_{M}-\bar{B}\right) \cdot 1 \\
= & {[w(B)-\operatorname{sgn}(B) \cdot 1]-[w(\bar{B})-\operatorname{sgn} \bar{B} \cdot 1] . }
\end{aligned}
$$

From the diagram

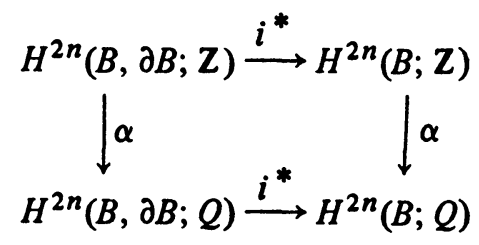

where $\alpha$ is the coefficient homomorphism, we see that $V$ contains a natural integral lattice $L=$ image $i^{*} \circ \alpha$ as well as its dual lattice $L^{+}=($image $\alpha) \cap V$. Since the homomorphism $\partial: W(Q) \rightarrow W(Q / Z)$ maps the kernel of sgn: $W(Q) \rightarrow$ $\mathbf{Z}$ isomorphically onto $W(Q / Z)$, the peripheral invariant $\operatorname{per}(M)$ may be identified with the induced finite form on $L^{+} / L$. To compute the peripheral invariant we relate it to a more familiar finite form on $M$.

For any closed oriented $M^{4 n-1}$ denote by $G$ the torsion subgroup of $H^{2 n}(M ; Z)$. If $x, y \in G$, then $x=\beta(z)$ for some $z \in H^{2 n-1}(M ; Q / Z)$ where $\beta$ is the Bockstein homomorphism for the coefficient sequence $0 \rightarrow \mathrm{Z} \rightarrow Q \rightarrow$ $Q / Z \rightarrow 0$. Using the pairing $\mathbf{Z} \times Q / Z \rightarrow Q / Z$, define $\lambda(x, y)=\langle z \cup y,[M]\rangle \in$ 
$Q / Z$. $\lambda$ is the linking form of $M$, a symmetric nonsingular finite form representing an element $L(M) \in W(Q / Z)$.

(2.1) THEOREM. If $M^{4 n-1}=\partial B^{4 n}$ as above then $\operatorname{per}(M)=-L(M)$ in $W(Q / Z)$.

The proof will be preceded by a lemma. Let $j: M \rightarrow B$ be the inclusion and define the following subgroups of $G$ :

and

$$
H=\left\{x \in G \mid x=j^{*}(\xi) \text { for some } \xi \in H^{2 n}(B ; Z)\right\} \text {, and }
$$

$$
K=\left\{x \in G \mid x=j^{*}(\xi) \text { for some } \xi \in \operatorname{Tor}\left(H^{2 n}(B ; \mathrm{Z})\right)\right\} .
$$

Applying Poincaré duality, one can show that $K$ is isomorphic to $G / H$.

(2.2) LEMMA. Under the linking form $(\lambda, G), H=\{x \in G \mid \lambda(x, y)=0$ for all $y \in K$ \}. That is, $H=K^{\perp}$.

Proof. First we show that $H \subseteq K^{\perp}$. Let $x \in K, y \in H$. In the diagram

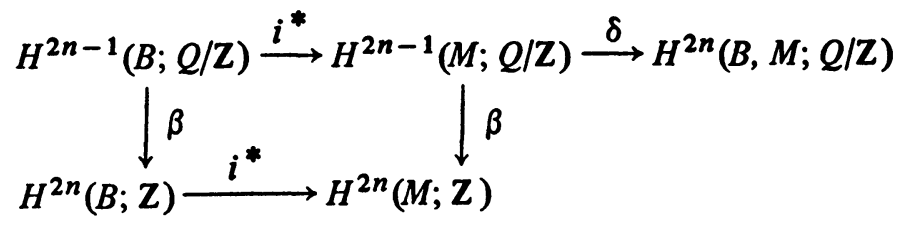

there exist $\eta, \xi \in H^{2 n}(B ; Z)$ with $i^{*}(\eta)=x, i^{*}(\xi)=y$ and $\mu \in H^{2 n-1}(B ; Q / Z)$ with $\beta(\mu)=x$. Then

$$
\begin{aligned}
\lambda(x, y) & =\left\langle i^{*}(\mu) \cup y,[M]\right\rangle=\left\langle i^{*}(\mu) \cup i^{*}(\xi),[M]\right\rangle \\
& =\left\langle\delta i^{*}(\mu) \cup \xi,[B, M]\right\rangle=0 .
\end{aligned}
$$

So $H \subseteq K^{\perp}$. But since $K \simeq G / H$ the nonsingularity of the form implies that $H=K^{\perp}$.

The result shows that the class in $W(Q / Z)$ represented by the linking form on $G$ may also be represented by the induced form on $H / K$.

Proof of (2.1). Note that the lattice $L$ may be identified via $\alpha$ with the image of $i^{*}: H^{2 n}(B, M ; Z) \rightarrow H^{2 n}(B ; Z) /$ Torsion. Also its dual lattice $L^{+}$ corresponds with the summand of $H^{2 n}(B ; Z) /$ Torsion containing $L$ as a subgroup of finite index. Thus we have an exact sequence

$$
0 \rightarrow L \rightarrow L^{+} \rightarrow H / K \rightarrow 0 .
$$


Now consider the following diagram

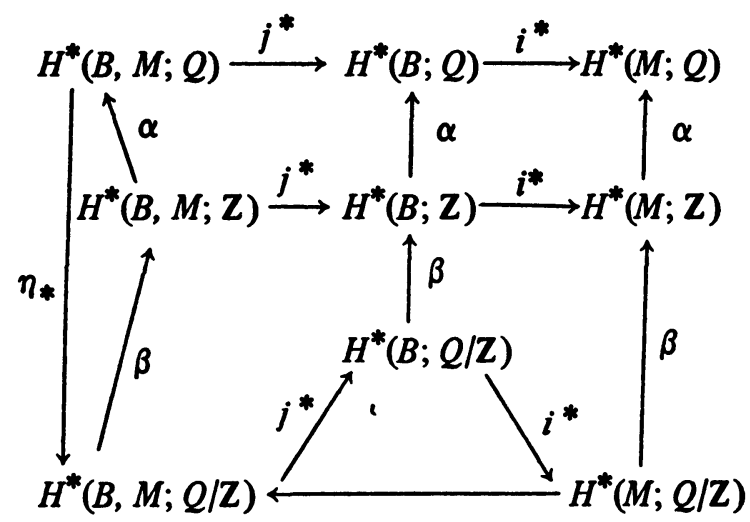

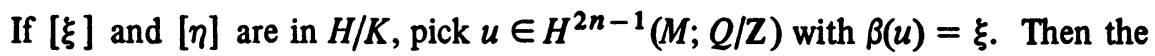
linking form on $G$ induces the form on $H / K$ given by

$$
\lambda([\xi],[\eta])=\langle u \cup \eta,[M]\rangle \text { in } Q / Z
$$

On the other hand, suppose $\xi=i^{*}(x)$ and $\eta=i^{*}(y)$. We know that $i^{*} \alpha(x)=0$ so let $z \in H^{2 n}(B, M ; Q)$ such that $j^{*}(z)=\alpha(x)$. The form $\delta(f)$ on $H / K$ may be given by

$$
\begin{aligned}
\delta(f)([\xi],[\eta]) & =\left\langle z \cup i^{*}(y),[B, M]\right\rangle \\
& =\left\langle\pi_{*}(z) \cup y,[B, M]\right\rangle \bmod \mathbf{Z} .
\end{aligned}
$$

Now $\lambda([\xi],[\eta])=\langle u \cup \eta,[M]\rangle=\langle\delta(u) \cup y,[B, M]\rangle$. So if we knew that $\pi_{*}(z)=-\delta(u)$, the two forms on $H / K$ would coincide and the theorem would follow. The fact that $\pi_{*}(z)=-\delta(u)$ may be established by representing the classes by cochains and chasing through the previous diagram.

(2.3) COROLLARY. The peripheral invariant, per $(M)$, may be defined for any closed oriented $(4 n-1)$-manifold and is an invariant of the oriented homotopy type of $M$.

If $M^{4 m}$ and $K^{4 k-1}$ are closed oriented manifolds then it may be checked that $\operatorname{per}\left(M^{4 m} \times K^{4 k-1}\right)=\operatorname{sgn}\left(M^{4 m}\right) \cdot \operatorname{per}\left(K^{4 k-1}\right)$. However, the natural extension of this statement to bundles is false. For example consider the Hopf fibration $S^{1} \rightarrow S^{3} \rightarrow S^{2}$ and suppose $T$ is a standard diffeomorphism of $S^{3}$ of period $p$ preserving the fibres. Then $S^{1} / T \rightarrow S^{3} / T \rightarrow S^{2}$ has $\operatorname{sgn}\left(S^{2}\right) \cdot \operatorname{per}\left(S^{1} / T\right)$ $=0$, but $\operatorname{per}\left(S^{2} / T\right) \neq 0$ as will be seen in the following examples. 
We now compute the peripheral invariant of a lens space. Let $T$ be the map of odd order $p$ on $S^{4 n-1}$ given by

$$
T\left(z_{1}, \ldots, z_{2 n}\right)=\left(\lambda^{r} z_{1}, \ldots, \lambda^{r} 2 n_{2 n}\right)
$$

where $\lambda=e^{2 \pi i / p}$ and $\left(r_{i}, p\right)=1$ for each $i$. The quotient $S^{4 n-1} / T$ is the lens space $L^{4 n-1}\left(p ; r_{1}, \ldots, r_{2 n}\right)$. The linking form on $L^{4 n-1}\left(p ; r_{1}, \ldots, r_{2 n}\right)$

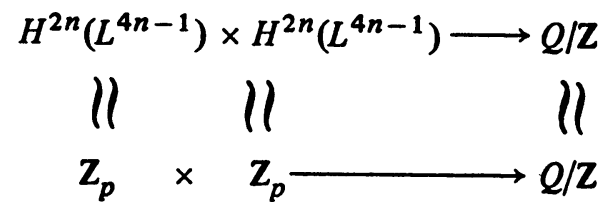

sends $(1,1) \rightarrow a / p$ for some integer $a$ prime to $p$.

(2.4) LEMMA. If $\alpha \in H^{2}(L) \simeq \mathrm{Z}_{p}$ is a generator and $\beta^{-1}(\alpha) \in H^{1}\left(L ; Z_{p}\right)$ is its pre-image under the Bockstein isomorphism

$$
\beta: H^{1}\left(L ; Z_{p}\right) \rightarrow H^{2}(L ; Z),
$$

then, when reduced $\bmod p,\left\langle\beta^{-1}(\alpha) \cdot \alpha^{2 n-1},[L]\right\rangle$ and a are equivalent modulo squares.

Proof. The element $1 \in Z_{p} \simeq H^{2 n}(L)$ is of the form $(K \alpha)^{n}$ for some integer $K \neq 0 \bmod p$. Let $b$ be a cochain in $S^{2 n-1}(L)$ such that $\delta(b)=p \cdot(K \alpha)^{n}$. Then from the definition of the linking form we have

$$
a=\left\langle b \cup(K \alpha)^{n},[L]\right\rangle \bmod p .
$$

Now let $K c \in S^{1}(L)$ be a cochain with $\delta(K c)=p \cdot K \alpha$. Then

$$
\delta\left(K c \cup(K \alpha)^{n-1}\right)=\delta(K c) \cup(K \alpha)^{n-1}=p \cdot(K \alpha)^{n} .
$$

So we may choose $b=K c \cup(K \alpha)^{n-1}$ and we have

$$
\begin{aligned}
a & =\left\langle K c \cup(K \alpha)^{n-1} \cup(K \alpha)^{n},[L]\right\rangle \bmod p \\
& =K^{2 n}\left\langle c \cup\left(\alpha^{2 n-1}\right),[L]\right\rangle \bmod p .
\end{aligned}
$$

Since $\delta(c)=p \alpha$, the mod $p$ reduction of $c$ is represented by $\beta^{-1}(\alpha)$ and the proof is complete.

This result may be used to establish that for the lens space $L^{4 n-1}(p ; 1, \ldots, 1)$ the linking form is $\langle 1\rangle_{p}$. The form for a general lens space may be computed by relating it to this standard model. For $L^{4 n-1}\left(p ; r_{1}, \ldots, r_{2 n}\right)$ let $l_{i}$ be an integer with $l_{i} r_{i}=1 \bmod p$ for $i=1, \ldots, 2 n$. View $S^{4 n-1}$ as the join of $2 n$ copies of $S^{1}, S^{4 n-1}=S^{1} * \cdots * S^{1}$.

We have two maps of period $p$ on $S^{4 n-1}: T_{1}$ in which the $i$ th coordinate is multiplied by $\lambda^{r_{i}}$ and $T_{2}$ in which each coordinate is multiplied by $\lambda$. Define 


$$
g:\left(S^{1} * \cdots * S^{1}, T_{1}\right) \longrightarrow\left(S^{1} * \cdots * S^{1}, T_{2}\right)
$$

to be the map which on the $i$ th coordinate is the standard map of degree $l_{i}$. Then $g$ is equivariant since

$$
\begin{aligned}
g\left(T_{1}\left(z_{1}, \ldots, z_{2 n}\right)\right) & =g\left(\lambda^{r_{1}} z_{1}, \ldots, \lambda^{r 2 n} z_{2 n}\right) \\
& =\left(\left(\lambda^{r_{1}} z_{1}\right)^{l_{1}}, \ldots,\left(\lambda^{r 2 n} z_{2 n}\right)^{l_{2 n}}\right) \\
& =\left(\lambda z_{1}^{l_{1}}, \ldots, \lambda z_{2 n}^{l_{2 n}}\right)=T_{2} g\left(z_{1}, \ldots, z_{2 n}\right) .
\end{aligned}
$$

If $l=l_{1} \cdot l_{2} \cdots l_{2 n}$, then $(l, p)=1$ and $g$ induces a map

$$
\bar{g}: L^{4 n-1}\left(p ; r_{1}, \ldots, r_{2 n}\right) \longrightarrow L^{4 n-1}(p ; 1, \ldots, 1)
$$

of degree $l$. It follows that the linking form on $L^{4 n-1}\left(p ; r_{1}, \ldots, r_{2 n}\right)$ is given by $\langle l\rangle_{p}=\left\langle r_{1} r_{2} \cdots r_{2 n}\right\rangle_{p}$.

III. Applications to periodic maps. Suppose $M^{4 n}$ is a closed oriented differentiable manifold and $T$ is an orientation preserving diffeomorphism of $M$ of odd prime period $p$. There is a nonsingular symmetric rational bilinear form on $H^{2 n}(M ; Q)$ given by $f(x, y)=p\langle x \cup y,[M]\rangle \in Q$ which is invariant under the action of $T^{*}$. With respect to this inner product, the averaging operator $T=$ $p^{-1}\left(1+T^{*}+T^{* 2}+\cdots+T^{* p-1}\right)$ is selfadjoint. Hence the image of $T$, the set of vectors fixed by $T^{*}$, is an orthogonal direct summand. Let $V$ be the subspace of vectors fixed by $T^{*}$, and denote by $w(T, M)$ the class in $W(Q)$ represented by the restriction of $f$ to $V$. Since the quotient map $\pi: M \rightarrow M / T$ induces a monomorphism $\pi^{*}: H^{2 n}(M / T ; Q) \rightarrow H^{2 n}(M ; Q)$ onto $V$, we denote the signature of $w(T, M)$ by $\operatorname{sgn}(M / T)$.

Conner and Raymond [8] associated to such a periodic map an element of $W(Q)$ given by

$$
q(T, M)=w(T, M)-\operatorname{sgn}(M / T) \cdot 1
$$

where $1 \in W(Q)$ is the unit. Note that $q(T, M)$ lies in the kernel of the signature homomorphism and so can be viewed as an element of $W(Q / Z) . q(T, M)$ is a cobordism invariant because if $(M, T)$ bounds an action on a compact oriented manifold $\left(W^{4 n+1}, \bar{T}\right)$ then the standard approach shows $V$ admits an invariant self-annihilating subspace whose dimension is $1 / 2 \operatorname{dim} V$. It follows that $w(T, M)$ $=0$ and $\operatorname{sgn}(M / T)=0$.

$q(T, M)$ also vanishes on fixed point free actions. In this case $M / T$ is a closed oriented manifold and $w(T, M)$ is Witt equivalent to the middle dimensional form on $M / T$ which is unimodular. Thus $w(T, M)=\operatorname{sgn}(M / T) \cdot 1$ and $q(T, M)=0$. 
Since $q(T, M)$ is a cobordism invariant that vanishes on fixed point free actions, we know that it is determined by the fixed point set $F \subseteq M$ and the representation of $\mathbf{Z}_{p}$ in its normal bundle.

We describe now another invariant defined in terms of the fixed set and depending on a systematic method for orienting its equivariant normal bundle. If $p \equiv 3 \bmod 4$ then -1 is not a square $\bmod p$ and we may give the normal bundle to $F$ a complex structure such that all eigenvalues of the action are of the form $e^{2 \pi i \beta / p}$ where $\beta$ is a square $\bmod p$. This complex structure on the normal bundle of $F$ together with the orientation for $M$ determines an orientation for $F$.

If $p \equiv 1 \bmod 4$ we orient the fixed set arbitrarily and divide it into two parts $F_{0}$ and $F_{1}$ as follows. For a given component of $F$, denote by $e^{2 \pi i \gamma_{1} / p}$, $\ldots, e^{2 \pi i \gamma_{k / p}}$ the eigenvalues of the action in the normal bundle. Then if $\gamma_{1}$. $\gamma_{2} \cdots \gamma_{k}$ is a square mod $p$ we put the component in $F_{0}$. If this product is not a square $\bmod p$ we put the component in $F_{1}$.

Define an element of $W(Q / Z)$ by

$$
\bar{q}(T, M)= \begin{cases}\operatorname{sgn} F \cdot\langle 1\rangle_{p} & \text { if } p \equiv 3 \bmod 4, \\ \operatorname{sgn} F_{0}\langle 1\rangle_{p}+\operatorname{sgn} F_{1}\langle\alpha\rangle_{p} & \text { if } p \equiv 1 \bmod 4\end{cases}
$$

where $\alpha$ is a nonsquare $\bmod p$. It is easy to see that $\bar{q}(T, M)$ vanishes if the action bounds or if the action is fixed point free.

Our main objective is to prove

(3.1) THEOREM. $q(T, M)=\bar{q}(T, M)$.

This was proved by Conner and Raymond for $p=3$ and for weakly complex involutions [8]. The general theorem for $p=2$ appears in [1]

Consider the periodic map on $\mathrm{CP}(2)$ given by $t\left[z_{0}, z_{1}, z_{2}\right]=\left[\lambda z_{0}, \lambda^{2} z_{1}, z_{2}\right]$ where $\lambda=e^{2 \pi i / p}$. There are three isolated fixed points at which the representations may be described by $\left(\lambda, \lambda^{-1}\right),\left(\lambda^{-1}, \lambda^{-2}\right)$ and $\left(\lambda, \lambda^{2}\right)$, and the action of $t^{*}$ in $H^{2}(\mathbf{C P}(2) ; Q)$ is the identity. In computing $q(T \times t, M \times \mathbf{C P}(2))$ the contribution from $H^{2 n+2}(M ; Q) \otimes H^{0}(\mathrm{CP}(2) ; Q)$ is clearly self-annihilating. A direct consequence is that $q(T \times t, M \times C P(2))=q(T, M)$ in $W(Q / Z)$.

For $p \equiv 3 \bmod 4$ the orientations of the fixed points of $t$ are either $(-,+,+)$ if 2 is a square $\bmod p$ or $(-,-,-)$ if 2 is not a square $\bmod p$. Hence the signature of the fixed set of $T \times t$ is either $\operatorname{sgn}(F)$ or $-3 \operatorname{sgn}(F)$. On the other hand for $p \equiv 1 \bmod 4,-1$ is a square $\bmod p$, so the parity of $\operatorname{sgn} F_{0}$ and $\operatorname{sgn} F_{1}$ remain unchanged. Therefore

$$
\bar{q}(T \times t, M \times \mathbf{C P}(2))=\bar{q}(T, M) .
$$


We conclude that in proving (3.1) we may assume that the codimension of $F$ in $M$ is arbitrarily large.

If $N$ denotes an equivariant closed tubular neighborhood of $F$ in $M$, we denote by $w(T, N)$ the element of $W(Q)$ given by the obvious inner product on the image of

$$
H^{2 n}(N / T, \partial N / T ; Q) \rightarrow H^{2 n}(N / T ; Q)
$$

and by $\operatorname{sgn}(T, N)$ its signature. Theorem 1.3 of [8] gives the following relation in $W(Q)$,

$$
\operatorname{per}(\partial N / T)=w(T, N)-\operatorname{sgn}(T, N) \cdot 1-q(T, M)
$$

Since we can assume that the codimension of the highest dimensional component of $F$ is greater than $2 n$, it would follow that $H^{2 n}(N / T ; Q) \simeq H^{2 n}(F ; Q)=0$ and hence that $q(T, M)=-\operatorname{per}(\partial N / T)$. We will have completed the proof of (3.1) if we can show

(3.2) Proposition. $-\operatorname{per}(\partial N / T)=\bar{q}(T, M)$.

Recall [7] that the action $(T, M)$ is equivariantly cobordant to an action in which the normal bundle to each component of the fixed set splits equivariantly into a sum of line bundles. So we assume this is true for $F$ and let $\hat{F}^{4 n-2 k}$ be a component of $F$ with normal bundle $\xi_{1} \oplus \cdots \oplus \xi_{k}$ where the action of $Z_{p}$ in $\xi_{r}$ is multiplication by $\lambda^{j_{r}}, 1 \leqslant r \leqslant k, \lambda=e^{2 \pi i / p}$. For each $r$ choose an integer $l_{r}$ with $l_{r} j_{r} \equiv 1(\bmod p)$.

Let $\xi_{r}^{l}=\xi_{r} \otimes \cdots \otimes \xi_{r}$ be the line bundle given by the $l_{r}$-fold tensor product and define an action of $Z_{p}$ on $\xi_{r}^{l}$ by multiplication by $\lambda$ in each fibre. For each $r$ there is a map of sphere bundles $\theta_{r}: S\left(\xi_{r}\right) \rightarrow S\left(\xi_{r}^{l}\right)$ given by $\theta_{r}(v)=$ $(v \otimes \cdots \otimes v)$. This map is equivariant since

$$
\begin{aligned}
\theta_{r}(T(v)) & =\theta_{r}\left(\lambda^{j_{r}}(v)\right)=\left(\lambda^{j_{r}}(v) \otimes \cdots \otimes \lambda^{j_{r}}(v)\right) \\
& =\left(\lambda^{j_{r}}\right)^{l_{r}}(v \otimes \cdots \otimes v)=\lambda(v \otimes \cdots \otimes v) \\
& =T\left(\theta_{r}(v)\right) .
\end{aligned}
$$

Note that on fibres the map $\theta_{r}$ is of degree $l_{r}$.

Now take the fibre join

$$
S\left(\xi_{1}\right) * S\left(\xi_{2}\right) * \cdots * S\left(\xi_{k}\right)=S\left(\bigoplus_{r} \xi_{r}\right)=\partial \hat{N}
$$

where $\hat{N}$ is an equivariant tubular neighborhood of $\hat{F}$. Similarly there is the fibre join 


$$
S\left(\xi_{1}^{l_{1}}\right) * \cdots * S\left(\xi_{k}^{l_{k}}\right)=S\left(\bigoplus_{r} \xi_{r}^{l_{r}}\right)=\partial N^{\prime}
$$

where $N^{\prime}$ is the appropriate disk bundle, and the join $\theta_{1} * \cdots * \theta_{k}$ gives an equivariant bundle map $\theta: \partial \hat{N} \rightarrow \partial N^{\prime}$. On each fibre the degree of $\theta$ is $l=$ $\Pi_{r=1}^{k} l_{r}$ while on the base it has degree 1 . A spectral sequence argument shows that $\theta: \partial \hat{N} \rightarrow \partial N^{\prime}$ has degree $l$ and hence so does the map on the quotient $\theta / T$ : $\partial \hat{N} / T \rightarrow \partial N^{\prime} / T$.

To understand how this relates the linking forms, first note that $W\left(Z_{p}\right)$ is a module over $W\left(Z_{(p)}\right)$ where $Z_{(p)}$ is the integers localized at $p$. The product sends $([V],[G])$ into the element $[V \otimes G]$ in $W\left(Z_{p}\right)$. If $(l, p)=1$ then $[\langle l\rangle]$ $\in W\left(\mathbf{Z}_{(p)}\right)$ and we will denote $[\langle l\rangle] \cdot[G]$ by $l \otimes[G]$. Note that $l \otimes\langle\beta\rangle_{p}=$ $\langle l \beta\rangle_{p}$.

Now $\theta / T$ gives a map of fibrations

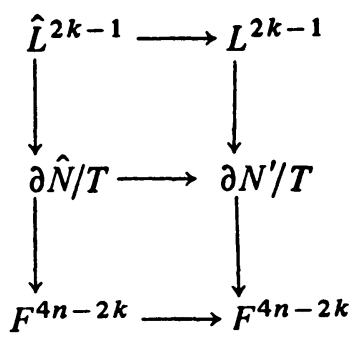

that is a $(2 k-2)$-equivalence. Since we can assume that $4 n-2 k<2 k-4$, it follows that $2 n<2 k-2$ and hence $(\theta / T)^{*}: H^{2 n}\left(\partial N^{\prime} / T ; Z\right) \rightarrow H^{2 n}(\partial \hat{N} / T ; Z)$ is an isomorphism. If $L^{\prime}$ and $\hat{L}$ denote the respective linking forms, we have

$$
\hat{L}\left((\theta / T)^{*}(x),(\theta / T)^{*}(y)\right)=l \cdot L^{\prime}(x, y) .
$$

Since $H^{2 n}\left(\partial N^{\prime} / T ; \mathrm{Z}\right)$ is all $p$-torsion, we have $\operatorname{per}(\partial \hat{N} / T)=l \otimes \operatorname{per}\left(\partial N^{\prime} / T\right)$.

We may now employ the technique of Conner and Raymond $[8, \S 2]$ to $\partial N^{\prime} / T$. Restated in terms of finite forms, they show that $\operatorname{per}\left(\partial N^{\prime} / T\right)=\operatorname{sgn} \hat{F} \cdot\langle 1\rangle_{p}$. Thus for the equivariant normal bundle $\hat{N}$ of $\hat{F}$ we conclude that $\operatorname{per}(\partial \hat{N} / T)=$ $\operatorname{sgn} \hat{F} \cdot\langle l\rangle_{p}$.

Recall the conventions for orienting the fixed set. For $p \equiv 3 \bmod 4,-1$ is not a square, so we choose a complex structure for the normal bundle in which all eigenvalues are of the form $\lambda^{r_{j}}$ where $r_{j}$ is a square $\bmod p$. Then $l$ is a square $\bmod p,\langle l\rangle_{p}=\langle 1\rangle_{p}$ and

$$
\operatorname{per}(\partial N / T)=\sum_{\hat{F}} \operatorname{sgn} \hat{F} \cdot\langle 1\rangle_{p}=\operatorname{sgn} F \cdot\langle 1\rangle_{p} .
$$

For $p \equiv 1 \bmod 4$, we orient in any way and place $\hat{F}$ in $F_{0}$ if $r_{1} \cdots r_{j}$ is a square $\bmod p$ and in $F_{1}$ if not. Then 


$$
\begin{aligned}
\operatorname{per}(\partial N / T) & =\sum_{\hat{F} \in F_{0}} \operatorname{sgn} \hat{F}\langle 1\rangle_{p}+\sum_{\hat{F} \in F_{1}} \operatorname{sgn} \hat{F} \cdot\langle\alpha\rangle_{p} \\
& =\operatorname{sgn} F_{0} \cdot\langle 1\rangle_{p}+\operatorname{sgn} F_{1} \cdot\langle\alpha\rangle_{p},
\end{aligned}
$$

where $\alpha$ is not a square $\bmod p$. These cases fully describe $\bar{q}(T, M)$ and we conclude that $q(T, M)=\bar{q}(T, M)$.

An immediate consequence of (3.1) is the following corollary.

(3.3) COROLlARY. If $(T, M)$ is as above and $T^{*}$ is the identity on $H^{2 n}(M ; Q)$ then

(a) for $p \equiv 3(\bmod 4)$, $\operatorname{sgn} M \equiv \operatorname{sgn} F(\bmod 4)$;

(b) for $p \equiv 1(\bmod 4), \operatorname{sgn} M \equiv \operatorname{sgn} F \equiv \operatorname{sgn} F_{0}(\bmod 2)$.

Proof. Since $T^{*}$ is the identity, $q(T, M)=\operatorname{sgn}(M) \cdot\langle 1\rangle_{p}$, and the conclusions follow from (3.1) and the structure of $W\left(Z_{p}\right)$.

Using different methods we have shown [5] that the conclusion in (3.3)(a) holds for all $T$ of odd period.

Before giving a final application we introduce another algebraic concept. Denote by $W\left(Z_{(2)}\right)$ the analogous Witt group for modules over $Z_{(2)}$, the integers localized at 2. If $(V, f)$ is such a form, there is an element $u \in V$ such that $f(u, v) \equiv f(v, v) \bmod 2$ for all $v \in V$. The class $u$ is a $W u$ class for $(V, f)$. Its existence follows from the fact that when tensored with $Z_{2}$, the form remains nonsingular and the function $v \rightarrow f(v, v)$ becomes a homomorphism into $Z_{2}$. The class $u$ is not unique, any other Wu class will be of the form $(u+2 w)$ for some $w \in V$; however, $f(u, u)$ is well defined $\bmod 8$ :

$$
\begin{aligned}
f(u+2 w, u+2 w) & =f(u, u)+4 f(w, w)+4 f(u, w) \\
& \equiv f(u, u)+8 f(w, w)(\bmod 8) .
\end{aligned}
$$

In the same manner as (1.5) there is a split short exact sequence

$$
0 \rightarrow W(\mathbf{Z}) \rightarrow W\left(\mathbf{Z}_{(2)}\right) \rightarrow \underset{p \text { odd prime }}{\bigoplus} W\left(\mathbf{Z}_{p}\right) \rightarrow 0 .
$$

Define a homomorphism $A: W\left(\mathbf{Z}_{(2)}\right) \rightarrow \mathbf{Z}_{8}$ by $A(V, f)=\operatorname{sgn} V-f(u, u)$ where $u$ is a Wu class for $(V, f)$.

(3.4) THEOREM ([9]). If $[V, f] \in W\left(\mathbf{Z}_{(2)}\right)$ lies in the image of $W(Z) \rightarrow$ $W\left(\mathrm{Z}_{(2)}\right)$ then sgn $V \equiv f(u, u) \bmod 8$.

It follows that the image of $W(\mathbf{Z})$ in $W\left(\mathbf{Z}_{(2)}\right)$ lies in the kernel of $A$. Hence there is an associated homomorphism $\tilde{A}$ and a commutative diagram 

$\bmod 8$.

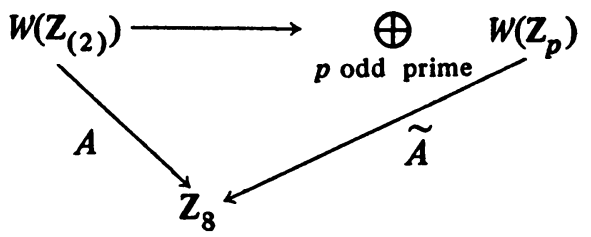

The value of $\tilde{A}$ on the various generators is given by

(3.5) Proposition. $\tilde{A}\langle 1\rangle_{p}=(1-p) \bmod 8$ and $\tilde{A}\langle\alpha\rangle_{p}=(5-p)$

Proof. It is clear that we can pull back $\langle 1\rangle_{p}$ to the form $\langle p\rangle$ in $W\left(Z_{(2)}\right)$, and that $A\langle p\rangle=(1-p) \bmod 8$. The form $\langle\alpha\rangle_{p}$ requires more care. We prove the result by induction on the prime $p$.

First for $p=3$ we may take $\alpha=-1$ so that $\langle\alpha\rangle_{3}$ pulls back to $\langle-3\rangle$ and $A\langle-3\rangle=-1+3=5-3(\bmod 8)$. This clearly will work for any $p \equiv 3 \bmod 4$.

Now let $p \geqslant 5$ be a prime, $p \equiv 1 \bmod 4$, and assume the result is true for all odd primes less than $p$. First we claim there is an odd prime $q<p$ which is a quadratic nonresidue mod $p$. If not then all odd integers less than $p$ as well as 0 are quadratic residues. But 4 is also a quadratic residue and this gives $(p+3) / 2$ quadratic residues, which is too many.

So let $q$ be a quadratic nonresidue $\bmod p, q$ an odd prime. Then $\langle 1\rangle_{p q}=$ $\langle p\rangle_{q}+\langle q\rangle_{p}$. Now $p \equiv 1 \bmod 4$ so by quadratic reciprocity $p$ is a quadratic nonresidue $\bmod q$. Hence

$$
\langle 1\rangle_{p q}=\langle\alpha\rangle_{q}+\langle\alpha\rangle_{p}
$$

and

$$
1-p q=\tilde{A}\langle 1\rangle_{p q}=\tilde{A}\langle\alpha\rangle_{q}+\tilde{A}\langle\alpha\rangle_{p} \equiv(5-q)+\tilde{A}\langle\alpha\rangle_{p} \bmod 8 .
$$

This gives

$$
\begin{aligned}
\tilde{A}\langle\alpha\rangle_{p} & \equiv 1-p q+q-5 \equiv 1-p q+q-p-5+p \\
& \equiv(1+q)(1-p)-(5-p) \bmod 8 .
\end{aligned}
$$

But $4 \mid(1-p)$ and $2 \mid(1+q)$; hence $\tilde{A}\langle\alpha\rangle_{p} \equiv-(5-p) \equiv 5-p \bmod 8$, because $5-p \equiv 0 \bmod 4$. This proves (3.5).

Now consider $A(w(T, M)-\operatorname{sgn}(M / T) \cdot 1)$. If $\bar{u}$ is a Wu class for the form on $H^{2 n}(M ; Q)$ then note that $\bar{u}+T^{*} \bar{u}+T^{* 2} \bar{u}+\cdots+T^{* p-1} \bar{u}$ is also a $W u$ class. Thus we may assume that the Wu class $u$ is fixed by $T^{*}$ and hence is also a Wu class for $V$. Therefore 


$$
\begin{aligned}
& =A(w(T, M))-\operatorname{sgn}(M / T) \cdot 1) \\
& =A(w(T, M))=\operatorname{sgn} V-f(u, u) \\
& =\operatorname{sgn}(M / T)-p\langle u, u\rangle \\
& \equiv \operatorname{sgn}(M / T)-p \operatorname{sgn} M \bmod 8 .
\end{aligned}
$$

On the other hand

$$
\tilde{A}(\bar{q}(T, M))=\left\{\begin{array}{l}
\operatorname{sgn} F \cdot \tilde{A}\langle 1\rangle_{p}, \quad p \equiv 3 \bmod 4 ; \\
\operatorname{sgn} F_{0} \tilde{A}\langle 1\rangle_{p}+\operatorname{sgn} F_{1} \tilde{A} \cdot\langle\alpha\rangle_{p}, \quad p \equiv 1 \bmod 4
\end{array}\right.
$$

Applying (3.1) and (3.5) we have

$$
\begin{aligned}
& \operatorname{sgn}(M / T)-p \operatorname{sgn} M \equiv \operatorname{sgn} F \cdot(1-p) \bmod 8, \quad p \equiv 3 \bmod 4 ; \\
& \operatorname{sgn}(M / T)-p \operatorname{sgn} M \equiv \operatorname{sgn} F_{0}(1-p)+\operatorname{sgn} F_{1}(5-p) \bmod 8, \quad p \equiv 1 \bmod 4 .
\end{aligned}
$$

Using the fact that $p^{2} \equiv 1 \bmod 8$ we may multiply the above congruences by $-p$ to yield a new proof of the following result of Conner [6].

(3.6) THEOREM.

$$
\operatorname{sgn} M-p \cdot \operatorname{sgn}(M / T) \equiv \begin{cases}\operatorname{sgn} F(1-p) \bmod 8 & \text { if } p \equiv 3 \bmod 4 \\ \operatorname{sgn} F_{1}(5-p) \bmod 8 & \text { if } p \equiv 1 \bmod 8 \\ \operatorname{sgn} F_{0}(1-p) & \text { if } p \equiv 5 \bmod 8 .\end{cases}
$$

\section{REFERENCES}

1. J. P. Alexander, $A W\left(Z_{2}\right)$ invariant for orientation preserving involutions, Proc. Amer. Math. Soc. 51 (1975), 455-460.

2. J. P. Alexander and G. C. Hamrick, The torsion G-signature theorem for odd order groups (in preparation).

3. J. P. Alexander, P. E. Conner, G. C. Hamrick and J. W. Vick, Witt classes of integral representations of an abelian p-group, Bull. Amer. Math. Soc. 80 (1974), 1179-1182.

4. J. P. Alexander, G. C. Hamrick and J. W. Vick, Bilinear forms and cyclic group actions, Bull. Amer. Math. Soc. 80 (1974), 730-734.

5. - The signature of the fixed set of a map of odd period, Proc. Amer. Math. Soc. (to appear).

6. P. E. Conner, Some applications of the $A-H-V$ formula, Semigroup Forum (to appear).

7. P. E. Conner and E. E. Floyd, Maps of odd period, Ann. of Math. (2) 84 (1966), 132-156. MR 34 \#3587.

8. P. E. Conner and F. Raymond, A quadratic form on the quotient of a periodic map, Semigroup Forum 7 (1974), 310-333.

9. J. Milnor and D. Husemoller, Symmetric bilinear forms, Ergebnisse der Mathematik, Springer-Verlag, Berlin and New York, 1973.

10. C. T. C. Wall, Quadratic forms on finite groups and related topics, Topology 2 (1963), 281-298. MR 28 \#133. 